\title{
Ungleiche Grundschulen und die meritokratische Fiktion im deutschen Schulsystem
}

\section{Georg Breidenstein}

Zusammenfassung Der Beitrag vertritt die These, dass Ungleichheit im Primarbereich ein Tabu darstellt, und zwar deshalb, weil sie meritokratisch nicht legitimierbar ist. Die Fiktion der Chancengleichheit erscheint zugleich notwendig, um die Selektion im Übergang zur Sekundarstufe rechtfertigen zu können. Denn die Verteilung von Schülerinnen und Schülern auf unterschiedlich aussichtsreiche Bildungsgänge im Sekundarbereich kann nur durch schulisch festgestellte und individuellen Kindern zugeschriebene Unterschiede im „Leistungsvermögen“ gerechtfertigt werden. Angesichts neuerer Befunde zur sozialräumlichen Segregation, zur elterlichen Schulwahl und zur Forcierung des Wettbewerbs im Bereich der Grundschule kann von gleichen Bildungschancen im Primarbereich allerdings kaum die Rede sein. Diese Einsicht ist prekär, sowohl für das Selbstverständnis der Grundschule als Institution als auch für die Legitimationsbasis des gegliederten Schulsystems insgesamt in Deutschland.

Schlüsselwörter Meritokratie $\cdot$ Schulische Selektion $\cdot$ Ungleichheit im Primarbereich 


\title{
Inequal primary schools and the fiction of meritocracy in the German school system
}

\begin{abstract}
The contribution discusses the connection between primary and secondary education in the German education system. With the transition to secondary education students are distributed to different tracks and this distribution is legitimized by achievement. This legitimation, which is the classic meritocratic one, relies on the idea of egalitarianism in primary education. But empirical evidence on residential segregation, on school choice and on marketization in German primary education points to the fact that equality is only a myth in primary education. This insight is precarious for the self-concept of primary school and for legitimacy of the school system as a whole in Germany.
\end{abstract}

Keywords Meritocracy · Tracking in the German school system · Inequality in primary education

Im folgenden Beitrag soll diskutiert werden, dass und warum ungleiche Bildungschancen im Primarbereich, im Sinne ungleicher Bedingungen zwischen verschiedenen Grundschulen, kaum thematisiert werden - weder von den handelnden Akteuren noch von der Grundschulpädagogik. Es wird die These vertreten, dass Ungleichheit im Primarbereich geradezu ein Tabu darstellt, und zwar deshalb, weil sie meritokratisch nicht legitimierbar ist. Im Gegenteil: Die Fiktion der Chancengleichheit ist, unabhängig von empirischer Evidenz, notwendig, um die Selektion im Übergang zur Sekundarstufe rechtfertigen zu können. Denn die Verteilung von Schülerinnen und Schülern auf unterschiedlich aussichtsreiche Bildungsgänge im Sekundarbereich kann nur durch schulisch festgestellte und individuellen Kindern zugeschriebene Unterschiede im „Leistungsvermögen“ gerechtfertigt werden.

Die Argumentation erfolgt in drei Schritten: Zunächst wird der systematische Stellenwert einer Grundschule „für alle Kinder“ für das selektive deutsche Schulsystem aufgezeigt. Im zweiten Schritt werden empirische Evidenzen dafür gesammelt, dass von gleichen Bildungschancen im Primarbereich kaum ausgegangen werden kann. Abschließend wird das angesprochene Tabu der Thematisierung dieser Ungleichheit im Primarbereich genauer diskutiert und zwar bezogen auf Akteure, Eltern und Schulen, sowie Grundschulforschung und Grundschulpädagogik. Mit ungleichen Grundschulen steht nicht nur das Selbstverständnis der Grundschule als Institution, sondern auch die Legitimationsbasis des selektiven deutschen Schulsystems zur Debatte.

\section{Die Bedeutung der Grundschule für das selektive deutsche Schulsystem}

In Deutschland werden Schülerinnen und Schüler in fast allen Bundesländern nach dem 4. Schuljahr auf unterschiedlich aussichtsreiche Bildungsgänge verteilt (in Berlin und Brandenburg nach dem 6. Schuljahr; in Mecklenburg-Vorpommern bilden 
die Klassen 5 und 6 eine „schulartenunabhängige Orientierungsstufe“). Das ist im weltweiten Vergleich nahezu singulär (von allen bei PISA teilnehmenden OECD Staaten wird nur in Österreich und in einigen Kantonen der Schweiz genauso früh selektiert), in den meisten Bildungssystemen reicht die gemeinsame Schulzeit bis zum 8. Schuljahr, oft auch bis zum Ende der Pflichtschulzeit. Das heißt nicht, dass es in diesen Bildungssystemen keine Bildungsungleichheit gäbe; bekanntermaßen gibt es z.B. in der angelsächsischen Bildungswelt gravierende Unterschiede zwischen einzelnen Schulen und auch zwischen ganzen Sektoren des Bildungssystems, etwa zwischen privaten und öffentlichen Schulen (Ball 2003; Walford 2006), aber es gibt nicht (oder später) die offizielle Verteilung der Schülerschaft auf unterschiedlich wertvolle Bildungsgänge. Die systematische hierarchische Stratifikation des Sekundarschulwesens ab Klasse 5 findet sich allein in den genannten deutschsprachigen Schulsystemen. Selbstverständlich werden Hauptschulen, Mittelschulen, Sekundarschulen oder Gemeinschaftsschulen nicht als ,minderwertig“ deklariert, aber es ist doch letztlich unbestreitbar, dass das Gymnasium mit dem Abitur den vielversprechenderen, vielseitigeren und wertvolleren Schulabschluss anzubieten hat. Zudem zeigt auch die empirische Bildungsforschung, dass die verschiedenen Schulformen der Sekundarstufe in Deutschland durchaus verschiedene „Lern- und Entwicklungsmilieus“ (Baumert et al. 2006) bereitstellen.

Gerechtfertigt wird diese ungleiche Verteilung von Bildungs- und Lebenschancen durch den Bezug auf „Leistungsunterschiede“, die individuellen Schülerinnen und Schülern zugeschrieben werden. Eine andere Legitimationsbasis für die Ungleichbehandlung von Schülern im Bildungssystem scheint in moderner Gesellschaft auch nicht denkbar. Man kann nicht auf den gesellschaftlichen Status der Eltern oder askriptive Merkmale wie Geschlechtszugehörigkeit rekurrieren, sondern muss Ungleichbehandlung in persönlichem Verdienst, sprich: „Leistung“, begründen (Becker und Hajdar 2017; Solga 2005). Dass die Verteilung der Schülerinnen und Schüler im Übergang auf die unterschiedlichen Bildungsgänge der Sekundarstufe in Deutschland empirisch betrachtet keineswegs ausschließlich nach „Leistung“ erfolgt, sondern mehrfachen und gravierenden ,Verzerrungen“ entsprechend der sozialen Herkunft unterliegen, ist inzwischen gut belegt und auch weithin thematisiert (Bos et al. 2007; Van Ackeren und Klemm 2019). - Dies ändert aber nichts daran, dass die legitimatorische Basis der Verteilung nur eine meritokratische sein kann. Die Legitimität der ausgesprochen frühen und scharfen Selektivität des deutschen Schulsystems mag zwar durch die Ergebnisse der empirischen ÜbergangsForschung gelitten haben, insgesamt aber scheint sie erstaunlich stabil. Die Forderung einer einheitlichen Sekundarstufe wird kaum erhoben, und das Gymnasium, als Schulform mit einem dezidierten Selektionsanspruch, scheint in der deutschen bildungspolitischen Diskussion nahezu sakrosankt.

Für die Aufrechterhaltung der Behauptung einer meritokratisch legitimierten Verteilung auf unterschiedlich aussichtsreiche Bildungsgänge im Übergang zur Sekundarstufe ist nun aber die Fiktion einer egalitären Primarstufe unabdingbar: Die Grundschule muss systematisch allen Kindern die gleichen Chancen geboten haben, damit Selektionsentscheidungen an deren Ende als gerechtfertigt gelten können. Diese Konstruktion gehört zum Gründungskontext der inzwischen 100-jährigen deutschen Grundschule: Ihr Auftrag in der jungen Demokratie bestand darin, die 
(vormoderne) Trennung in Volksschule und „Vorschulen“, die ausschließlich auf das Gymnasium vorbereiten, zu ersetzen durch ,,die für alle gemeinsame Grundschule, auf der sich auch das mittlere und höhere Schulwesen aufbaut“ (Reichsgrundschulgesetz 1920, § 1). Einer solchen „Etablierung der gemeinsamen Grundschule als Sockel eines im Anschluss daran gegliederten Schulsystems“ (Van Ackeren und Klemm 2019, S. 402) war, in der Formulierung von Friedeburgs (1989, S. 218), „von vorneherein die Last der Selektion auf den Weg gegeben“. In der Grundschule wird weitgehend über Schullaufbahnen von Kindern entschieden.

Systematisch und schultheoretisch trägt die Grundschule diese Last - ob und inwiefern sie sich dadurch als Institution tatsächlich belastet fühlt erscheint interessanter Weise weniger klar. Götz und Sandfuchs (2011, S. 43) beklagen zwar „die dauerhafte Diskrepanz zwischen dem Selbstverständnis der Grundschule als primär fördernder Schulstufe, die individuelle und gruppenspezifische Startnachteile ausgleicht, und dem tatsächlich nachgewiesenen Selektionseffekt, der historisch wie aktuell eine Benachteiligung von Grundschülern aus unteren sozialen Schichten belegt“‘. Damit bestätigen sie allerdings letztlich ein Selbstverständnis der Grundschule, in dem ihr Selektionsauftrag keine prominente Rolle zu spielen scheint - gepflegt wird eher der Identitätskern der „Schule für alle Kinder“, der einzigen nicht-selektiven Schulform im deutschen Schulsystem. ${ }^{1}$ In aktuellen Einführungen in die Grundschulpädagogik findet sich durchgängig die Pflege dieses „Mythos“ (Götz 2020), Reflexionen zur funktionalen Bedeutung der Grundschule für das selektive deutsche Schulsystem sucht man vergebens. An anderer Stelle habe ich gezeigt, wie die Sortierung von Schülerinnen und Schülern nach „Leistung“ im gesamten (deutschsprachigen) schulpädagogischen Diskurs in Gestalt des kanonischen Theorems der „Selektionsfunktion“ externalisiert und der „Gesellschaft“ zugerechnet wird (Breidenstein 2018). Verkannt bzw. verleugnet wird dabei, dass die Selektion zunächst und insbesondere am Übergang von der Primarstufe zur Sekundarstufe ausschließlich im Rahmen des Erziehungssystems geschieht.

\section{Ungleiche Grundschulen}

Diese Grundkonstruktion im deutschen Schulsystem, die sich bis zum Weimarer „Schulkompromiss“ zurückverfolgen lässt, gerät nun massiv ins Wanken, sobald das Gleichheits-Postulat für die Grundschule in Frage steht. Empirisch scheint klar, dass von gleichen Bedingungen für alle Grundschulen und alle Grundschülerinnen und Grundschüler in Deutschland kaum die Rede sein kann. Das zeigen in eindrucksvoller Weise Forschungen zur sozialräumlichen Segregation von Einzugsgebieten und Schülerschaften (Ditton und Krüsken 2007), die hier nur knapp angesprochen werden, verwiesen sei ansonsten auf die ausgezeichnete Darstellung von Parade und Heinzel (2020). Die soziale Segregation deutscher (Groß-) Städte wirkt sich über die

\footnotetext{
1 Dass dies nie zugetroffen hat und auch das oben zitierte Reichsgesetz schon im nächsten Satz einschränkt: ,auf Hilfsschulklassen findet diese Bestimmung keine Anwendung“, wurde im Selbstverständnis der deutschen Grundschule eher abgeblendet, was vermutlich im Rahmen jener (Selbst-) Verortung der Grundschule im selektiven deutschen Schulsystem, die hier zur Diskussion steht, unabdingbar war und ist.
} 
Einzugsgebiete von Grundschulen unmittelbar auf die soziale Zusammensetzung der Schülerschaften aus; sozial homogene Schulbezirke bilden sich in sozial homogenen Schülerschaften von Grundschulen ab. Eine aktuelle Studie von Helbig und Jähnen (2018) zeigt die Zunahme sozialer Segregation in vielen deutschen Städten, wobei insbesondere Familien mit Kindern von der „sozialen Spaltung“ deutscher Städte betroffen sind. In Einzelfallstudien erweist sich die Festlegung der Schulbezirke durchaus als Politikum: Insbesondere bei Neuschneidungen von Einzugsgebieten kann es zu Auseinandersetzungen um die Zuordnung einzelner Wohngebiete oder Straßen zu bestimmten Grundschulen kommen. - Hier spielt offensichtlich nicht nur die pragmatische Erreichbarkeit eines Schulstandortes eine Rolle, sondern auch die gewünschte bzw. zu vermeidendende soziale Zusammensetzung der Schülerschaft (Breidenstein und Voigt 2020; Radtke und Stosic 2009).

Die erhebliche soziale Selektivität im Zugang zu Grundschulen schlägt sich in sehr unterschiedlichen Übergangsquoten auf das Gymnasium nieder; darauf hat Hauf (2007) schon vergleichsweise früh aufmerksam gemacht. Schräpler und Weishaupt (2019) zeigen in einer detaillierten Untersuchung zur Entwicklung in NRW, dass sich die Übergangsquoten auf das Gymnasium zwischen sozialräumlich differenten Clustern von Grundschulen erheblich unterscheiden. Auch in einer Stadt wie Halle/ Saale können Übergangsquoten einzelner Grundschulen auf das Gymnasium zwischen 13 und $85 \%$ variieren (Breidenstein und Voigt 2020). Vor dem Hintergrund insgesamt in den letzten Jahrzehnten gestiegener Bildungserwartungen von Eltern scheint in manchen Grundschulen in eher bürgerlichen Wohnvierteln der Übergang zum Gymnasium der „Normalfall“ geworden zu sein, während in vielen Grundschulen in sozial benachteiligten Wohnvierteln in der Stadt oder in eher ländlichen Regionen immer noch nur eine Minderheit der Kinder auf das Gymnasium wechselt (Schräpler und Weishaupt 2019; Jeworutzki und Schräpler 2019).

Unterschiede zwischen Grundschulen, die durch sozialräumliche Kontexte bedingt sind, scheinen sich aktuell durch Tendenzen der Steigerung des Wettbewerbs zwischen Grundschulen und durch die Ausweitung von Schulwahlaktivitäten von Eltern im Zugang zur Grundschule noch zu verstärken (Krüger et al. 2020). Aufforderungen zur Schulprofilierung werden in Städten wie Berlin schon länger vor dem Hintergrund von Segregationstendenzen diskutiert (Fincke und Lange 2012; Flitner 2007). Dabei scheinen Grundschulen in schwierigeren Wohnquartieren in der Ressourcenausstattung (Unterrichtsversorgung, Qualifikation der Lehrkräfte) noch zusätzlich benachteiligt zu sein (Helbig und Nikolai 2019). Schulwahlaktvitäten von Eltern knüpfen hier an, nehmen Unterschiede zwischen Grundschulen wahr und verstärken diese tendenziell noch. „So zeigt sich, dass Eltern und hier in erster Linie ressourcenstarke Eltern aus der Mittelschicht, Möglichkeiten finden, trotz eingeschränkter, aber in allen Bundesländern prinzipiell vorhandener Wahlmöglichkeiten aktiv eine öffentliche Grundschule zu wählen“ (Stirner et al. 2019, S. 442). Eine solche Wahl wird z.B. in Form von Gastschulanträgen oder Umzügen (oder fingierten Umzügen) realisiert, wenn die eigentliche Einzugsgebietsschule vermieden werden soll; aber auch die Entscheidung für die Einzugsgebietsschule wird oft als zu verantwortende und zu begründende „Wahl“" verstanden (Roch 2020).

Zusätzliche Optionen elterlicher Schulwahl eröffnen Privatschulen, die insbesondere im Primarbereich einen wahren Boom erleben (Koinzer und Mayer 2015). 
Das Privatschulwesen mit seinen verschiedenen Milieus ist differenziert zu betrachten (Ullrich und Strunck 2012), und es macht sicher einen Unterschied, ob eine Schulgründung auf dem Land den Wegfall der staatlichen Grundschule kompensiert oder ein zusätzliches, womöglich kostspieliges Angebot im großstädtischen Raum darstellt. Aber insgesamt ist zu konstatieren, dass Grundschulen in privater Trägerschaft zu Ungleichheit im Primarbereich beitragen, auch wenn Stirner et al. (2019, S. 451) festhalten, ,dass Privatschulen nicht als hauptursächlich für die schulische Segregation in der Primarstufe anzusehen sind; sie haben vielmehr einen moderierenden, verstärkenden Effekt. In erster Linie ist die schulische Segregation durch die residentielle Segregation bedingt“.

Die angesprochenen Schulwahlambitionen und -aktivitäten werden in der Bildungsforschung inzwischen als „Parentokratie“ diskutiert (Waldow 2014), denn hier werden aussichtsreichere, erfolgversprechendere Positionen im Bildungssystem nicht nach „Leistung“ (meritokratisch), sondern nach den Ressourcen der Eltern vergeben. Dabei geht es nicht nur um finanzielle Ressourcen, sondern auch um den Zugang zu Informationen, oder um die Investition von Zeit und Engagement. Dabei ist es nun wichtig, zwischen dem Übergang von der Primarstufe zur Sekundarstufe und dem Übergang vom Elementar- zum Primarbereich systematisch zu unterscheiden. Ersterer, der Wechsel in die Sekundarstufe mit ihren unterschiedlichen Bildungsgängen, wird in der empirischen Bildungsforschung schon lange und unter ungleichheitskritischen Vorzeichen untersucht - beim Übergang in die Primarstufe stellt sich das ganz anders dar.

Der in Deutschland immense Einfluss der sozialen Herkunft auf die wegweisende Bildungsentscheidung am Eingang der Sekundarstufe wird durchgängig kritisch gesehen. Ob man eher mit Bezug auf Boudon ,primäre“ und „,sekundäre“ Effekte sozialer Herkunft untersucht (Becker und Hadjar 2017) oder mit Bourdieu den Einfluss des familialen Habitus auf die Schulkarriere zu bestimmen sucht (Kramer et al. 2009), der enge Zusammenhang zwischen dem Herkunftsmilieu und der Schullaufbahn gilt als ,ungerecht“. Dabei steht im Hintergrund der kritischen Diskussion dieses Zusammenhangs letztlich die Fiktion einer „meritokratischen“ Verteilung von Schülerinnen und Schülern auf unterschiedlich aussichtsreiche Bildungsgänge. Eine meritokratische, also „leistungsgerechte“ Verteilung wird in dieser Perspektive durch den Einfluss der sozialen Herkunft ,,verzerrt“" oder , überformt“", aber sie ist nicht außer Kraft gesetzt. Vermutlich wird die Idee einer meritokratischen Verteilung von Schülerinnen und Schülern im Übergang zur Sekundarstufe durch die ungleichheitskritische Bildungsforschung implizit sogar gestärkt, denn sie bildet die Folie und den Maßstab der Kritik. Das Ausmaß der Einflussnahme der sozialen Herkunft wird in der Regel bestimmt über den Vergleich von Bildungsentscheidungen (bzw. ihrer Wahrscheinlichkeit) mit Daten aus den Schulleistungsstudien: Als ungerecht, nicht legitimierbar, gelten demnach insbesondere jene Wahrscheinlichkeiten eines Gymnasialbesuchs, die nicht durch einen gemessenen Leistungsvorsprung gedeckt sind (Baumert et al. 2006). Die „Leistung“ der 10 jährigen Schülerinnen und Schüler erscheint in dieser Forschungslinie als objektiv messbar und sie erhält in den Studien zu Bildungsungleichheit die starke Position eines Maßes für Ungerechtigkeit.

Während also im Übergang zur Sekundarstufe die Idee, dass Leistungsunterschiede der Schülerinnen und Schüler der Ungleichbehandlung zugrunde liegen könnten 
bzw. sollten, nicht zuletzt in der ungleichheitskritischen Bildungsforschung eine zentrale Rolle spielt, erscheint diese Idee im Zugang zu unterschiedlichen Grundschulen nicht sinnvoll. Unterschiedliche Noten, unterschiedliche Kompetenzen am Ende der Grundschulzeit können als unterschiedliche schulische „Leistungen“ von Schülerinnen und Schülern verstanden werden (die in meritokratischer Logik auch unterschiedliche Chancen rechtfertigen können) - im Übergang zur Grundschule gibt es noch keine „Leistungen“ in diesem Sinne. Es gibt zwar Schuleingangsuntersuchungen und diverse Tests zu Lernständen und Lernvoraussetzungen, und sie dienen auch in gewissem Umfang als Grundlage von Selektionsentscheidungen, z. B. für die Bildung von ,Sprachlernklassen“, Zurückstellungen oder die Einschulung in eine Förderschule (Bildungsbericht 2018), aber diese Tests können jedenfalls nicht „meritokratisch“ verwendet werden.

Die meritokratische Legitimation von Ungleichheit verlangt zwingend die Idee einer Leistung, die dem Individuum als ,Verdienst“ zugerechnet werden kann (Nerowski 2018) - dies erscheint bei Kindern, die noch vor der Einschulung stehen, nicht sinnvoll. ${ }^{2}$ Der Grundschule kommt die unverzichtbare Aufgabe zu, schulische Leistungsunterschiede $\mathrm{zu}$ erzeugen und zu konstatieren, die dann als Legitimation für Selektionsentscheidungen im Übergang zum gegliederten Sekundarschulwesen dienen können. Man kann sich diese Funktion verdeutlichen, wenn man sich vorstellt, sechsjährige Kinder würden gleich bei der Einschulung auf stratifizierte Bildungsgänge verteilt - etwa in Abhängigkeit von mitgebrachten Lernvoraussetzungen. Dies erscheint nicht meritokratisch legitimierbar, denn solcherart Unterschiede könnten eben nicht als „Verdienst“ des individuellen Kindes verstanden werden.

„Leistung“, die dem individuellen Kind, seinen Fähigkeiten, seiner Anstrengungsbereitschaft etc. zugeschrieben werden kann, wird erst im Verlauf der Grundschule eingeführt. Hess (2020) etwa beschreibt in einer ethnographischen Studie, wie Grundschulkinder im Verlauf des zweiten Schuljahres mit der Praxis einer Leistungsbewertung vertraut gemacht werden, die den Vergleich von Leistungssubjekten impliziert, Leistungen in Form von Noten markiert und individuellen Kindern zurechnet. Aber auch in reformpädagogischen „Alternativen“ zu Zensuren werden Fähigkeiten zugeschrieben und festgestellt, die letztlich Übergangsentscheidungen legitimieren.

\footnotetext{
2 Das Problem der „Leistungsgerechtigkeit“ ist durchaus brisant im schulpädagogischen Diskurs. Dies zeigt die aktuelle Debatte in der Zeitschrift für Erziehungswissenschaft, wo auf den Beitrag von Nerowski eine Kritik durch Berkemeyer (2020) erfolgte und dann wiederum eine Replik Nerowskis (2020) sowie schließlich eine „Metakritik“ durch Tenorth (2020). Diese Debatte muss hier nicht im Detail referiert werden, denn auch hier wird die systematische Bedeutung des Primarbereichs für die Legitimation von Ungleichheit kaum berücksichtigt. Hingewiesen sei aber darauf, dass auch Nerowski (2018), der sich, mit guten Gründen, um eine gerechtigkeitstheoretische Begründung für ungleiche Bildungszertifikate bemüht, ein so genanntes „Bildungsminimum“ für alle Schülerinnen und Schüler konzipiert, das er allerdings nicht institutionell der Grundschule zuweist.
} 


\section{Zur Thematisierung und Tabuisierung von Ungleichheit im Primarbereich}

Im Zugang zur Primarstufe gibt es also keine Möglichkeit, ungleiche Bildungschancen meritokratisch zu legitimieren (Bloch et al. 2019). Doch das Narrativ der Meritokratie ist in moderner Gesellschaft die einzige Möglichkeit (Bildungs-) Ungleichheit als legitim auszuweisen. Ungleichheit im Primarbereich darf es mithin nicht geben. Sie stellt, das ist die These des Beitrags, ein Tabu dar, weil es keine Möglichkeit der Legitimation gibt. Ungleichheit darf es im Primarbereich aber nicht nur deshalb nicht geben, weil eine Selektion nach Leistung im Zugang zur Grundschule nicht denkbar erscheint, sondern auch deshalb, weil die Fiktion gleicher Bildungschancen „für alle Kinder“ im Primarbereich - wie oben bereits argumentiert wurde - für die Fiktion einer meritokratischen Verteilung von Bildungschancen im Übergang zum Sekundarbereich unverzichtbar ist. Mit der Fiktion der Bildungsgleichheit im Primarbereich steht und fällt die Rechtfertigung der Selektion im Sekundarbereich des deutschen Bildungssystems. Alle Kinder müssen, das ist die systematische Voraussetzung, die gleichen Startchancen gehabt haben, wenn man Ungleichheit im Übergang zur nächsten Stufe des Bildungssystems deren „Leistung“ zurechnen will. - Die postulierte und systemisch notwendige Voraussetzung gleicher Bildungschancen für alle Kinder in der ersten Schule ist allerdings vor der empirischen Evidenz der ungleichen Chancen zu schützen.

Die Tabuisierung von Ungleichheit im Primarbereich lässt sich empirisch auf unterschiedlichen Ebenen beobachten - sowohl in Interviews mit schulwahlaktiven Eltern als auch in Interviews mit Schulleitungen. Die schulwahlaktiven Eltern, die das Sample unserer qualitativen Untersuchung bildeten (Krüger et al. 2020), zeigten im Interview durchaus ein deutliches Bewusstsein von dem Problem sozialer Segregation im Primarbereich. Indem sie sich selbst zu den „Bildungsnahen“ oder „besser Gestellten“ rechneten, wären sie wohl zu den potentiellen Gewinnern von Segregationseffekten zu zählen. Die Affirmation von sozialen Absetzbewegungen mit dem Mittel der Schulwahl finden wir allerdings in keinem einzigen Interview die Orientierung an der Idee der Chancengleichheit im Primarbereich scheint dies in jedem Fall zu verbieten. Beobachten lässt sich in den Elterninterviews einerseits das Dementi von Segregationseffekten, oder, andererseits, der Rekurs auf Elternverantwortung und die Orientierung am Wohl des eigenen Kindes. Damit scheint eine Möglichkeit gefunden, die eigentlich unverzichtbare Orientierung an Egalität zu relativieren, sich selbst zu positionieren und Schulwahl im Primarbereich zu legitimieren (Breidenstein et al. 2020). Auch Schulleitungen dementieren im Interview die Idee eines „Wettbewerbs“ zwischen Grundschulen, der diese in Konkurrenz zueinander stellen würde und ggf. mit Segregationseffekten einhergehen würde. Die Schulleiterinnen, einschließlich Privatschulen, weisen die Orientierung an Markt und Konkurrenz zurück und verweisen (stattdessen) auf die Idee der „Schulvielfalt“ und die „Passung“ unterschiedlicher Schulprofile zu unterschiedlichen Elternwünschen - auf diese Weise scheinen Unterschiede zwischen Grundschulen legitimierbar zu werden (Breidenstein et al. 2015).

$\mathrm{Zu}$ fragen bleibt aber auch nach der Rolle von (Grund-) Schulpädagogik und (Grund-) Schulforschung: (Wie) wird Ungleichheit hier zum Thema gemacht? Das 
Problem der sozialen Selektivität im deutschen Bildungssystem wird systematisch im Übergang zum Sekundarschulwesen verortet. Becker (2016, S. 8) etwa schreibt bündig: „In der soziologischen Bildungsforschung wird in der Regel die soziale Ungleichheit von Bildungschancen am Ende der Grundschulzeit untersucht. Denn der Übergang von der Grundschule auf die weiterführenden Schulen ist nicht nur die erste, sondern auch die entscheidende Weichenstellung von Bildungslaufbahnen im deutschen Schulsystem“. Diese Bemerkung ist so treffend wie symptomatisch, denn Becker, dessen Beitrag dem Thema der „Chancengleichheit bei der Einschulung und in der Primarstufe“ gewidmet ist, behandelt „die Grundschule“ durchgängig terminologisch und konzeptionell als eine einheitliche Institution. Er interessiert sich durchaus für Ungleichheiten zwischen Kindern, die diese (aus dem Vorschulbereich) in „die Grundschule“ mitbringen und die „,kaum durch die Grundschule ausgeglichen werden können, aber für Bildungschancen am Ende der Primarstufe mit ausschlaggebend sind“" (Becker 2016, S. 15), Unterschiede zwischen Grundschulen jedoch und somit Ungleichheit, die im Primarbereich selbst erzeugt würde, werden in keiner Weise zum Thema.

Dieses Muster der Thematisierung von „Ungleichheit“ in Bezug auf „die Grundschule" findet sich nahezu durchgängig. Etwas zugespitzt: Ungleich sind die Kinder, die in die Schule kommen (in ihren Lernvoraussetzungen, ihrer Motivation, ihrem Sprachvermögen etc.), während „die Grundschule“ sich redlich bemüht (mit mehr oder weniger Erfolg), mit dieser Ungleichheit zurecht zu kommen oder sie nach Möglichkeit abzubauen (Klemm 2008; Ramseger und Wagener 2008). - Der ganze Diskurs um die „Heterogenität“ der (Grundschul-) Kinder folgt diesem Muster: Ungleich sind die Kinder, die Grundschule versucht dieser Ungleichheit gerecht zu werden. Dies ist dort, wo sich Grundschulpädagogik und Grundschulforschung auf pädagogisches und didaktisches Handeln richten, plausibel: Hier steht tatsächlich die Heterogenität der Lerngruppe als didaktische Herausforderung im Vordergrund. Durch eine solche Thematisierung, die Ungleichheit als Problem perspektiviert, das von außen an die Institution Grundschule herangetragen wird, gerät allerdings jene Ungleichheit nicht in den Blick, die im und durch den Primarbereich selbst erzeugt wird: die ungleichen Lernchancen, die ungleiche Grundschulen bieten. Letztlich trägt also der (grund-) schulpädagogische Diskurs um „Heterogenität“ (Budde et al. 2015; Trautmann und Wischer 2011) zur Entthematisierung von jener Ungleichheit bei, die systematisch im Primarbereich selbst erzeugt wird. ${ }^{3}$ Wie gesagt: Dass sie - als Institution - Bildungsungleichheit (re-) produziert passt einerseits wenig zum Selbstbild der Grundschule als „Schule für alle Kinder“, andererseits würde diese Einsicht die meritokratische Legitimationsbasis des deutschen Schulsystems insgesamt erschüttern.

\footnotetext{
${ }^{3}$ Idel (2016, S. 104) macht darauf aufmerksam, dass der Rekurs auf die „Heterogenität“ der Kinder als Begründung für die „Individualisierung“ des Unterrichts auch mit Blick auf die Reflexion des Unterrichtshandelns nicht unproblematisch ist: „Das Dilemma der wohlgemeinten Individualisierung von Unterricht ist darin zu sehen, dass sie die meritokratische Gerechtigkeitsordnung durch das aufgezeigte Muster einer differenzsensiblen individualisierten Zuschreibung von selbst verantworteter Leistung verschärft und damit die Beteiligung der Schule bzw. des Unterrichts und letztlich des Handelns der Lehrkräfte an der Reproduktion von Bildungsungleichheit aus dem Blick zu geraten droht.“”
} 
Nun könnte man einwenden, dass es empirisch fraglich ist, ob Ungleichheiten, die aus unterschiedlichen familialen und sozialen Kontexten in die Grundschule „mitgebracht“" werden, im Primarbereich lediglich stabilisiert und fortgeschrieben oder insgesamt verstärkt werden. ${ }^{4}$ Möglicherweise gelingt sogar in gewissem Umfang der Ausgleich von ungleichen Eingangsvoraussetzungen im Primarbereich. Zu der Frage, ob der Primarbereich selbst eher ungleichheitsverstärkend oder -abschwächend wirkt, fehlt, wenn ich das richtig sehe, tatsächlich ein belastbarer Forschungsstand. Die Unterschiedlichkeit der Kontextbedingungen für einzelne Grundschulen (entsprechend der sozialräumlichen Segregation, s. oben) ist zwar inzwischen in den Blick genommen, und dass die ungleichen Grundschulen auch Unterschiede verstärken, insofern sie unterschiedliche „Lern- und Entwicklungsmilieus“ bereitstellen, ist zwar wahrscheinlich, aber es mangelt an empirischer Evidenz zu den tatsächlichen Effekten. Dabei wäre es nicht nur bildungssoziologisch, sondern auch bildungspolitisch wichtig, den Beitrag der Grundschule zur (Re-) Produktion sozialer Ungleichheit im Verhältnis zu familialen Bedingungen einschätzen zu können (Downey und Condron 2016). Gegenüber diesem Einwand, der auf die mangelnde Empirie zu dem eigenständigen Anteil des Primarbereichs in der Generierung sozialer Ungleichheiten verweist, kann einerseits die Vermutung entwickelt werden, dass dieses Forschungsdefizit genau ein Ausdruck der Tabuisierung von Ungleichheit im Primarbereich sein könnte. Zum anderen aber ist festzuhalten, dass es aufgrund der vorliegenden, oben knapp referierten, Evidenzen als äußerst unwahrscheinlich erscheint, dass es der Grundschule gelingt, tatsächlich gleiche Chancen für alle Kinder im Bildungssystem zu schaffen. Die schulische Zuschreibung und Zertifizierung von „Leistungen“ gegenüber individuellen Kindern, die die Grundschule für das selektive deutsche Schulsystem übernimmt, kann also kaum (im meritokratischen Sinn) ,gerecht“ sein. - Dieser Zusammenhang ist von dem Tabu betroffen.

Funding Open Access funding provided by Projekt DEAL.

Open Access Dieser Artikel wird unter der Creative Commons Namensnennung 4.0 International Lizenz veröffentlicht, welche die Nutzung, Vervielfältigung, Bearbeitung, Verbreitung und Wiedergabe in jeglichem Medium und Format erlaubt, sofern Sie den/die ursprünglichen Autor(en) und die Quelle ordnungsgemäß nennen, einen Link zur Creative Commons Lizenz beifügen und angeben, ob Änderungen vorgenommen wurden.

Die in diesem Artikel enthaltenen Bilder und sonstiges Drittmaterial unterliegen ebenfalls der genannten Creative Commons Lizenz, sofern sich aus der Abbildungslegende nichts anderes ergibt. Sofern das betreffende Material nicht unter der genannten Creative Commons Lizenz steht und die betreffende Handlung nicht nach gesetzlichen Vorschriften erlaubt ist, ist für die oben aufgeführten Weiterverwendungen des Materials die Einwilligung des jeweiligen Rechteinhabers einzuholen.

Weitere Details zur Lizenz entnehmen Sie bitte der Lizenzinformation auf http://creativecommons.org/ licenses/by/4.0/deed.de.

\footnotetext{
${ }^{4}$ Ich bin den Gutachter*innen der ZfG dankbar für diesen Einwand, denn er gibt mir Gelegenheit, die Argumentation an dieser Stelle zu klären.
} 


\section{Literatur}

Van Ackeren, I., \& Klemm, K. (2019). 100 Jahre Grundschule - Soziale Chancenungleichheit und kein Ende. Zeitschrift für Grundschulforschung, 12(2), 399-414.

Autorengruppe Bildungsberichterstattung (2018). Bildung in Deutschland 2018 (zit. als Bildungsbericht 2018). https://www.bildungsbericht.de/de/bildungsberichte-seit-2006/bildungsbericht-2018/ pdf-bildungsbericht-2018/bildungsbericht-2018.pdf. Zugegriffen: 26.03.2020.

Ball, S. J. (2003). Class strategies and the education market. The middle classes and social advantage. London: Routledge.

Baumert, J., Stanat, P., \& Watermann, R. (2006). Schulstruktur und die Entstehung diffenzieller Lern- und Entwicklungsmilieus. In J. Baumert, P. Stanat \& R. Watermann (Hrsg.), Herkunftsbedingte Disparitäten im Bildungswesen: Differenzielle Bildungsprozesse und Probleme der Verteilungsgerechtigkeit. Vertiefende Analysen im Rahmen von PISA 2000 (S. 95-188). Wiesbaden: VS.

Becker, R. (2016). Chancengleichheit bei der Einschulung und in der Primarstufe. Theoretische Überlegungen und empirische Evidenzen. Zeitschrift für Grundschulforschung, 9(1), 7-19.

Becker, R., \& Hadjar, A. (2017). Meritokratie - Zur gesellschaftlichen Legitimation ungleicher Bildungs-, Erwerbs- und Einkommenschancen in modernen Gesellschaften. In R. Becker (Hrsg.), Lehrbuch der Bildungssoziologie (3. Aufl. S. 37-62). Wiesbaden: Springer VS.

Berkemeyer, N. (2020). Über die Schwierigkeit, das Leistungsprinzip im Schulsystem gerechtigkeitstheoretisch zu begründen. Replik auf Christian Nerowski. Zeitschrift für Erziehungswissenschaft, 21, 447-464.

Bloch, R., Breidenstein, G., Krüger, J. O., Mader, M., \& Winter, D. (2019). Die prekäre Legitimierung des limitierten Zugangs zu exklusiven Bildungseinrichtungen. In W. Helsper, H.-H. Krüger \& J. Lüdemann (Hrsg.), Exklusive Bildung und neue Ungleichheit. 65. Beiheft der Zeitschrift für Pädagogik (S. 267-276). Weinheim, Basel: Beltz Juventa.

Bos, W., Schwippert, K., \& Stubbe, T.C. (2007). Die Kopplung von sozialer Herkunft und sozialer Schülerleistung im internationalen Vergleich. In W. Bos, et al. (Hrsg.), IGLU 2006 Lesekompetenzen von Grundschulkindern in Deutschland im internationalen Vergleich (S. 225-247). Münster: Waxmann.

Breidenstein, G. (2018). Das Theorem der „Selektionsfunktion der Schule“ und die Praxis der Leistungsbewertung. In S. Reh \& N. Ricken (Hrsg.), Leistung als Paradigma (S. 307-327). Wiesbaden: Springer VS.

Breidenstein, G., \& Voigt, T. (2020). Ein Schulbezirk als Politikum. In J. O. Krüger, A. Roch \& G. Breidenstein (Hrsg.), Szenarien der Grundschulwahl. Eine Untersuchung von Entscheidungsdiskursen am Übergang zum Primarbereich (S. 71-92). Wiesbaden: Springer VS.

Breidenstein, G., Krüger, J.-O., \& Roch, A. (2020). Schulwahl als Elitebildung? Zur Bearbeitung des Segregationsverdachtes im elterlichen Diskurs. In J. O. Krüger, A. Roch \& G. Breidenstein (Hrsg.), Szenarien der Grundschulwahl. Eine Untersuchung von Entscheidungsdiskursen am Übergang zum Primarbereich (S. 41-56). Wiesbaden: Springer VS.

Breidenstein, G., Krüger, J.-O., Tyagunova, T., \& Böckelmann, K. (2015). Von Schullandschaften zu Schulmärkten? Ambivalenzen im Diskurs zur Grundschulwahl. Zeitschrift für Grundschulforschung. Bildung im Elementar- und Primarbereich, 8(2), 42-54.

Budde, J., Blasse, N., Bossen, A., \& Rißler, G. (Hrsg.). (2015). Heterogenitätsforschung. Empirische und theoretische Perspektiven. Weinheim: Beltz Juventa.

Ditton, H., \& Krüsken, J. (2007). Sozialräumliche Segregation und schulische Entwicklung. Diskurs Kindheits- und Jugendforschung, 2(1), 23-38.

Downey, D., \& Condron, D. (2016). Fifty years since the Coleman report: rethinking the relationship between schools and inequality. Sociology of Education, 89, 207-220.

Fincke, G., \& Lange, S. (2012). Segregation an Grundschulen: Der Einfluss der elterlichen Schulwahl. Berlin: Sachverständigenrat deutscher Stiftungen für Integration und Migration. https://www.svrmigration.de/wp-content/uploads/2014/11/Segregation_an_Grundschulen_SVR-FB_WEB.pdf. Zugegriffen: 26.03.2020.

Flitner, E. (2007). Schöne Schulprofile. Zur Dynamik ethnischer Segregationsprozesse am Beispiel der Entwicklung exklusiver Angebote im Berliner öffentlichen Schulsystem 1995-2005. In R. . Casale (Hrsg.), Bildung und Öffentlichkeit. Jürgen Oelkers zum 60. Geburtstag. Weinheim: Beltz.

von Friedeburg, L. (1989). Bildungsreform in Deutschland. Geschichte und gesellschaftlicher Widerspruch. Frankfurt a.M.: Suhrkamp.

Götz, M. (2020). Die Geschichtsschreibung zur Grundschule - eine Mythenpflege? In N. Böhme, B. Dreer, H. Hahn, S. Heinecke, G. Mannhaupt \& S. Tänzer (Hrsg.), Mythen, Widersprüche und Gewissheiten. 
Eine wissenschaftliche Bestandsaufnahme nach 100 Jahren Grundschule. Jahrbuch Grundschulforschung. Wiesbaden: Springer VS.

Götz, M., \& Sandfuchs, U. (2011). Geschichte der Grundschule. In W. Einsiedler, M. Götz, A. Hartinger, F. Heinzel, J. Kahlert \& U. Sandfuchs (Hrsg.), Handbuch Grundschulpädagogik und Grundschuldidaktik (S. 32-44). Bad Heilbrunn: Klinkhardt.

Hauf, T. (2007). Innerstädtische Bildungsdisparitäten an der Übergangsschwelle von den Grundschulen zum Sekundarschulsystem. Zeitschrift für Pädagogik, 53(3), 299-313.

Helbig, M., \& Jähnen, S. (2018). Wie brüchig ist die soziale Architektur unserer Städte? Trends und Analysen der Segregation in 74 deutschen Städten. WZB-discussion paper, Bd. P 2018-001. Berlin: Wissenschaftszentrum Berlin für Sozialforschung.

Helbig, M., \& Nikolai, R. (2019). Bekommen die sozial benachteiligsten Schüler*innen die „,besten“ Schulen? Eine explorative Studie über den Zusammenhang von Schulqualität und sozialer Zusammensetzung von Schulen am Beispiel Berlins. Discussion Paper, Bd. P-2019-002. Berlin: Wissenschaftszentrum Berlin für Sozialforschung. https://bibliothek.wzb.eu/pdf/2019/p19-002.pdf.

Hess, A. (2020). Disziplin und Leistung im Alltag einer zweiten Grundschulklasse. Eine ethnographische Studie. Wiesbaden: Springer VS.

Idel, T.-S. (2016). Individualisierung und Differenz. Ein Konzept für mehr Bildungsgerechtigkeit? In K. Rabenstein \& B. Wischer (Hrsg.), Individualisierung schulischen Lernens. Mythos oder Königsweg? (S. 93-108). Seelze: Kallmeyer.

Jeworutzki, S., \& Schräpler, J.-P. (2019). Strukturwandel gleich Bildungswandel? Auswirkungen des Strukturwandels auf die Bildungschancen der Kinder im Ruhrgebiet. Standort, 43, 107-113.

Klemm, K. (2008). Vierzig Jahre Chancenungleichheit in der Grundschule - keine Hoffnung auf Abhilfe in Sicht? In J. Ramseger \& M. Wagener (Hrsg.), Chancenungleichheit in der Grundschule. Ursachen und Wege aus der Krise (S. 17-23). Wiesbaden: Springer VS.

Koinzer, T., \& Mayer, T. (2015). Private Schulen - Entwicklung und empirische Befunde unter besonderer Berücksichtigung des Grundschulwesens. Zeitschrift für Grundschulforschung, 8(2), 28-41.

Kramer, R.-T., Helsper, W., Thiersch, S., \& Ziems, C. (2009). Selektion und Schulkarriere. Kindliche Orientierungsrahmen beim Übergang in die Sekundarstufe I. Wiesbaden: VS.

Krüger, J. O., Roch, A., \& Breidenstein, G. (2020). Szenarien der Grundschulwahl. Eine Untersuchung von Entscheidungsdiskursen am Übergang zum Primarbereich. Wiesbaden: Springer VS.

Nerowski, C. (2018). Leistung als Kriterium von Bildungsgerechtigkeit. Zeitschrift für Erziehungswissenschaft, 21(3), 441-464.

Nerowski, C. (2020). Zur Relevanz sprachlicher Präzision für die Rede über Schule, Leistung und Gerechtigkeit. Eine Antwort auf Nils Berkemeyer. Zeitschrift für Erziehungswissenschaft, 23, 455-459.

Parade, R., \& Heinzel, F. (2020). Sozialräumliche Segregation und Bildungsungleichheiten in der Grundschule - eine Bestandsaufnahme. Zeitschrift für Grundschulforschung, 2.

Radtke, F.-O., \& Stosic, P. (2009). Lokale Bildungsräume: Ansatzpunkte für eine integrative Schulentwicklung. geographische revue, 1/2009, 34-51.

Ramseger, J., \& Wagener, M. (Hrsg.). (2008). Chancenungleichheit in der Grundschule. Ursachen und Wege aus der Krise. Wiesbaden: Springer VS.

Roch, A. (2020). erantwortungsvoll einschulen! Die „Qual der Grundschulwahl“ als Performanz ,guter“ Elternschaft? In J. O. Krüger, A. Roch \& G. Breidenstein (Hrsg.), Szenarien der Grundschulwahl. Eine Untersuchung von Entscheidungsdiskursen am Übergang zum Primarbereich (S. 191-220). Wiesbaden: Springer VS.

Schräpler, J.-P., \& Weishaupt, H. (2019). Grundschule und sozialräumliche Ungleichheit. Zeitschrift für Grundschulforschung, 12(2), 415-438.

Solga, H. (2005). Meritokratie - die moderne Legitimation ungleicher Bildungschancen. In P. A. Berger \& H. Kahlert (Hrsg.), Institutionalisierte Ungleichheiten? Stabilität und Wandel von Bildungschancen (S. 19-38). Weinheim: Juventa.

Stirner, P., Hoffmann, L., Mayer, T., \& Koinzer, T. (2019). Eine gemeinsame Grundschule für alle? Die Grundschule als Ort sozio-ökonomischer Ungleichheit und Segregation. Zeitschrift für Grundschulforschung, 12(2), 439-455.

Tenorth, H.-E. (2020). Über die Schwierigkeiten der Pädagogik, über Leistung und Gerechtigkeit im Schulsystem zu reden. Eine Metakritik zu Berkemeyers Nerowski-Kritik. Zeitschrift für Erziehungswissenschaft (ZfE), 23, 439-449.

Trautmann, M., \& Wischer, B. (2011). Heterogenität in der Schule. Eine kritische Einführung. Wiesbaden: VS. 
Ullrich, H., \& Strunck, S. (2012). Private Schulen in Deutschland - Entwicklungen und Diskurse. In H. Ullrich \& S. Strunck (Hrsg.), Private Schulen in Deutschland. Entwicklungen, Profile, Kontroversen (S. 11-25). Wiesbaden: VS.

Waldow, F. (2014). Von der Meritokratie zur Parentokratie? Zeitschrift für Erziehungswissenschaft, 17(S3), 43-58.

Walford, G. (2006). Markets and equity in education. Londen, New York: continuum. 